\title{
A remark on Fox's paper on shape \\ by
}

D. M. Hyman (New Brunswick, N. J.)

1. Introduction. Recently, R. H. Fox [2] has extended Borsuk's notion of "fundamental homotopy" from the category of compact metric spaces to general categories by considering equivalence classes of inverse systems under. appropriate equivalence relations. Specializing his construction to the category of metric spaces, Fox defines the "shape" of a metric space $X$ to be equivalence class of the inverse system of all neighborhoods of $X$ in an $A X R$ that contains $X$ as a closed subset; this is independent of the choice of $A$ NR, provided that $X$ is closed in it. For noncompact $X$, the restriction of closedness is sometimes inconvenient (see [2], footnote 6). The purpose of this note is to show that this restriction can be removed. Our main result is stated below; pertinent terms vre defined in the next section.

THeOREM 1. Let $X$ be a metric space. Suppose that $Y_{1}$ and $Y_{2}$ are ANR's such that $X \subset Y_{1}$ and $X \subset Y_{2}$. Then the inverse systems $U\left(X, Y_{1}\right)$ and $U\left(X, Y_{2}\right)$ are of the same similarity type.

2. Inverse systems. We begin by recalling some basic definitions from [2]. Let $\varepsilon$ be a category and let $\simeq$ be a compositive equivalence relation on the morphisms of $\varepsilon$. All categories considered in this section are assumed to be subcategories of $\delta$. Two morphisms in $\delta$ are concurrent if they hare the same domain and same range. If $e_{1}, e_{2}$ are concurrent and if $e$ is a morphism such that $e_{1} e \simeq e_{2} e$, then $e$ is an equalizer of $e_{1}$ and $e_{2}$.

Let $U$ be a category, with morphisms denoted by $\boldsymbol{u}$. If $U_{1}, U_{2}$ are objects of $U$, then $U_{1}$ precedes $U_{2}$ in $U$ if there exists a morphism in $U$ with domain $\bar{\zeta}_{1}$ and range. $U_{2}$. We say that $U$ is an inverse system if

(i) any two objects in $U$ have a common predecessor in $\boldsymbol{U}$, and

(ii) any two concurrent morphisms in $U$ have an equalizer in $U$.

For our application, the most important example of an inverse system is the collection $U=U(X, Y)$ of all neighborhoods of a subset $X$ of a metric space $Y ; \boldsymbol{u}$ is the set of all possible inclusion maps. Here, $\mathcal{E}$ is the topological category and $\simeq$ denotes homotopy. 
If $\boldsymbol{U}, \boldsymbol{V}$ are inverse systems, then a mutation $f: \boldsymbol{U} \rightarrow \boldsymbol{V}$ is any collection (in \&) of morphisms $f: U \rightarrow V$ from objects $U \in U$ to objects $V \in V$ such that

(i) if $u \in \boldsymbol{u}, f \in f, v \in \boldsymbol{v}$ and $v f u$ is defined, then $v f u \in f$;

(ii) every object of $\boldsymbol{V}$ is the range of some morphism in $f$; and

(iii) any two concurrent morphisms in $f$ have an equalizer in $U$.

If $f: \boldsymbol{U} \rightarrow \boldsymbol{V}$ and $\boldsymbol{g}: \boldsymbol{V} \rightarrow \boldsymbol{W}$ are mutations, their composition $\boldsymbol{g} f$ is the collection of all morphisms $g f, g \in g, f \in f$, that are defined. In particular, the collection ' $\boldsymbol{u}$ of all morphisms of $\boldsymbol{U}$ is a mutation from $\boldsymbol{U}$ to itself and acts as an identity under composition: $\boldsymbol{f} \boldsymbol{u}=\boldsymbol{f}$ and $\boldsymbol{u} \boldsymbol{g}=\boldsymbol{g}$ whenever these compositions are defined.

Two mutation $f, g: U \rightarrow V$ are similar (written $f \simeq g$ ) if concurrent morphisms $f \epsilon f, g \in g$ always have an equalizer in $U$. Two inverse systems $\boldsymbol{U}$ and $\boldsymbol{V}$ are of the same similarity type (written $\boldsymbol{U} \simeq \boldsymbol{V}$ ) if there exist mutations $f: U \rightarrow V, g: V \rightarrow U$ such that $g f \simeq \boldsymbol{u}$ and $f g \simeq \boldsymbol{v}$.

LEMMA 1 (Fox [2]). If $X$ is a closed subset of ANR's $Z_{1}$ and $Z_{2}$, then $\boldsymbol{U}\left(X, Z_{1}\right) \simeq \boldsymbol{U}\left(X, Z_{2}\right)$.

Completing this list of definitions, we say that an inverse system $\boldsymbol{V}$ is coinitial in an inverse system $U$ if

(i) $\boldsymbol{V}$ is a subcategory of $\boldsymbol{U}$;

(ii) each object of $U$ is preceded in $U$ by some object of $V$; and

(iii) any two concurrent morphisms in $U$ whose common domain is in $\boldsymbol{V}$ have an equalizer in $\boldsymbol{V}$. $\boldsymbol{V} \simeq \boldsymbol{U}$

th follows from Theorem 2.12 of [2] that if $V$ is coinitial in $U$, then

3. The inverse system $V(X, Z)$. Suppose that $X$ is a subset of a metric space $Y$, and let $\Psi$ denote the set of all continuous functions from $Y$ into $[0,1]$ with positive values in $X$. Let

$$
Z=Y \times[0,1]-((Y-X) \times\{0\}),
$$

and identify $X$ with $X \times\{0\} \subset Z$. Observe that $X$ is closed in $Z$. For each $U \in \boldsymbol{U}(X, Y)$ and each $\psi \in \Psi$, let

$$
\nabla(U, \psi)=\{(y, t) \in Z \mid y \in U \text { and } t<\psi(y)\} .
$$

It is clear that $V(U, \psi)$ is a neighborhood of $X$ in $Z$, that is, $V(U, \psi)$ $\epsilon U(X, Z)$. The collection of all such sets $V(U, \psi)$, together with all possible inclusion maps, is an inverse system which we denote by $\boldsymbol{V}(X, Z)$.

LEMTia 2. $V(X, Z) \simeq U(X, Z)$.

Proof. For brevity, write $U=U(X, Z)$ and $V=V(X, Z)$. To prove that $U \simeq \boldsymbol{V}$ it is sufficient to show that $\boldsymbol{V}$ is coinitial in $\boldsymbol{U}$, which, because the morphisms in $\boldsymbol{U}$ and $\boldsymbol{V}$ are inclusions, is equivalent to showing that each $U \in U$ contains some $V \in V$.
Let $d$ be a metric on $I$ bounded by 1 , and define a metric $\delta$ on $Y \times[0,1] \mathrm{by}$

$$
\delta\left(\left(y_{1}, t_{1}\right),\left(y_{2}, t_{2}\right)\right)=\max \left\{d\left(y_{1}, y_{2}\right),\left|t_{1}-t_{2}\right|\right\} .
$$

Now let $U \in \boldsymbol{U}$ be given, and let $W \subset Y \times[0,1]$ be an open set such that $U=W \frown Z$. Define a map $\psi \in \Psi$ by

$$
\psi(y)=\delta((y, 0), Y \times[0,1]-W) .
$$

It follows from (1) that

$$
\{(y, t) \in Y \times[0,1] \mid t<\psi(y)\} \subset W .
$$

Intersecting both sides of (2) with $Z$ gives

$$
\nabla(Y, \psi) \subset U
$$

which proves the lemma.

Lemma 3. $\boldsymbol{U}(X, Y) \simeq \boldsymbol{V}(X, Z)$.

Proof. For brevity, write $U=U(X, Y)$ and $V=V(X, Z)$. Define a mutation $f: U \rightarrow V$ as follows: If $U \in U, V \in V$, and $\varphi \in \Psi$ are such that the graph of $q \mid U$ lies in $V$, that is,

$$
\{(y, \varphi(y)) \mid y \in O\} \subset \nabla,
$$

then we define a map $f=f(U, V, \varphi): U \rightarrow \nabla$ by

$$
f(y)=(y, \varphi(y)) \quad \text { for all } y \in U .
$$

The collection of all such maps $f$ is a mutation $f: U \rightarrow V$. Now define a mutation $g: V \rightarrow U$ as follows: Let $\pi: Z \rightarrow Y$ denote the restriction to $Z$ of the coordinate projection $Y \times[0,1] \rightarrow Y$. If $U \in U$ and $V \in V$ satisfy $\pi(V) \subset U$, then we define a map $g=g(U, V): V \rightarrow U$ by

$$
g(z)=x(z) \text { for all } z \in \nabla .
$$

The collection of all such maps $g$ is a mutation $g: V \rightarrow U$. Then $g f \simeq u$; in fact, $g f=u$, for if $f$ and $g$ are of the form (1) and (2) and if $g f$ is defined, then $g f$ is just the inclusion of the domain of $f$ into the range of $g$.

To complete the proof of the Lemma, we must show that $f \boldsymbol{g} \simeq \boldsymbol{v}$. Let $f g \in f g$, where $g=g\left(T, \nabla_{1}\right)$ and $f=f\left(U, \nabla_{2}, \varphi\right)$. Then for $i=1,2$, there exist sets $W_{i} \in U(X, Y)$ and maps $\psi_{i} \in \Psi$ such that $T_{i}=T_{i}\left(W_{i}, \psi_{i}\right)$. Define a map $\psi: I \rightarrow[0,1]$ by

$$
\psi(y)=\min \left(\psi_{1}(y), \psi_{2}(y)\right) \quad \text { for all } y \in Y,
$$

and define a homotopy $h_{s}: \nabla\left(W_{1}, \psi\right) \rightarrow V_{2}$ by

$$
h_{s}(w, t)=(w,(1-s) t+s \cdot \varphi(w)) \quad \text { for all }(w, t) \in V\left(\Pi_{1}, \psi\right), 0 \leqslant s \leqslant 1 \text {. }
$$


Then $h_{0}$ is the inclusion of $V\left(W_{1}, \psi\right)$ into $V_{2}$, and $h_{1}$ is the restriction of $f g$ to $\boldsymbol{V}\left(W_{1}, \psi\right)$. This proves that $f \boldsymbol{g} \simeq \boldsymbol{v}$.

4. Proof of Theorem 1. For $i=1,2$, let $Z_{i}=Z_{i}\left(X, Y_{i}\right)$ be the set $Y_{i} \times[0,1]-\left(\left(Y_{i}-X\right) \times\{0\}\right)$ of $\S 3$. Because similarity is transitive, the theorem will be proved if we can establish the following sequence of similarities:

$$
U\left(X, Y_{1}\right) \simeq V\left(X, Z_{1}\right) \simeq U\left(X, Z_{1}\right) \simeq U\left(X, Z_{2}\right) \simeq V\left(X, Z_{2}\right) \simeq U\left(X, Y_{2}\right) .
$$

The first and last similarities follow from Lemma 3 ; the second and fourth follow from Lemma 2. The middle one is a consequence of Lemma 1 and the following result, which is based on a method of Fox [1].

LEMUMA 4. If $Y$ is an ANR and $X \subset Y$, then $Z(X, Y)$ is an ANR.

Proof. Write $Z=Z(X, Y)$. Let $A$ be a closed subset of a metric space $B$, and suppose $f: A \rightarrow Z$ is a map. Because $Y \times[0,1]$ is an ANR, there exists a neighborhood $W$ of $A$ in $B$ and an extension $g: W \rightarrow Y \times$ $\times[0,1]$ of $f$. Let $\lambda: W \rightarrow[0,1]$ be a map such that $\lambda^{-1}(0)=A$. Letting $\pi_{1}: Y \times[0,1] \rightarrow Y$ and $\pi_{2}: Y \times[0,1] \rightarrow[0,1]$. denote the coordinate projections, define a map $F: W \rightarrow Z$ by

$$
F(w)=\left(\pi_{1} g(w), \min \left\{1, \pi_{2} g(w)+\lambda(w)\right\}\right) \text { for all } w \in W .
$$

Then $F(W)$ is indeed contained in $Z$, and clearly $F$ extends $f$; hence $Z$ is an ANR.

We close by reformulating the results of the last two sections in the following manner, which might be of independent interest.

THEовем 2. If $X$ is a subset of a metric space $Y$, then there exists a metric space $Z$ containing $X$ as a closed subset such that the systems $U(X, Y)$ and $U(X, Z)$ are of the same similarity type; if $\bar{Y}$ is an $\mathrm{ANR}$, we may choose $Z$ to be an ANR.

\section{References}

[1] R. H. Fox, A characterization of absolute neighborhood retracts, Bull. Amer. Math. Soc. 48 (1942), pp. 271-275.

[2] - On shape, Fund. Math. 74 (1972), pp. 47-71

RUTGERS UNIVERSTYY, DOUGLASS COLIEGE

New Brunswick, New Jersey

Reçu par la Rédaction le 31. 8. 197Q

\section{Topological completeness of first countable Hausdorff spaces $I^{*}$}

\section{by}

H. H. Wicke (Athens, Ohio) and J. M. Worrell, Jr. (Albuquerque, N. Mex.)

1. Introduction. This paper is the first of several which present a theory of topological completeness for first countable Hausdorff spaces. The completeness concept introduced here, called basic completeness, permits the development of a theory analogous in many respect to that elaborated classically for complete metric spaces. In this connection it should be noted that a metrizable space is basically complete if and only if it has a topology-preserving metric in which it is complete $\left(^{1}\right)$. This article presents certain definitions and some set-theoretical and topological lemmas, which are both fundamental for the theory and have wider applicability, and proves certain characterization theorems.

One of the principal results proved here is that a Hausdorff space is an open continuous image of a complete metric space if and only if it is a basically complete space. It should be emphasized that regularity is not assumed. This theorem leads to the result that the class of basically complete spaces is the intersection of all classes $\mathrm{C}$ of Hausdorff spaces such that 1) $\mathrm{C}$ includes all metrically topologically complete spaces and 2) $\mathrm{C}$ is closed with respect to the application of open continuous mappings with Hausdorff images.

For the purposes of indicating the scope of the present results and of providing a basis for further discussion in subsequent papers we list here certain criteria for topological completeness. These are formulated in terms of two classes $\mathscr{B}$ and $\mathcal{C}$ of topological spaces. The members of $\mathscr{B}$ are subject to some uniformization condition $\left(^{2}\right)$ and $\mathcal{C}$ is a subclass of $\mathcal{B}$

* This work was supported by the United States Atomic Energy Commission. A preliminary version was given as a colloquium lecture at the University of New Mexico in May 1968.

(i) Such metrizable spaces will be called metrically topologically complete in accord with a standard usage.

(2) Uniformization condition and topological uniformization, as employed here, do not necessarily connote for the spaces to which they are applied the presence of a topology-preserving uniformity in the sense of A. Weil's definition. 